\title{
Estudio etnográfico de jóvenes skater: prácticas corporales y urbanas
}

\author{
Ethnographic study of young skaters: body and urban practices
}

\author{
* Ciria Margarita Salazar C., *Emilio Gerzaín Manzo Lozano, \\ *Aideé Consuelo Arellano Ceballos, *Lenin Tlamatini Barajas Pineda, ${ }^{* *}$ Anahy Valdivia
}

Salazar C., C. M., Manzo L., E. C., Arellano C., A. C., Barajas P., L. T., \& Valdivia, A. (2020). Estudio etnográfico de jóvenes skater: prácticas corporales y urbanas. Revista Ciencias de la Actividad Física UCM, № 21(2), julio-diciembre, 1-12. DOI: http://doi.org/10.29035/rcaf.21.2.6

\begin{abstract}
RESUMEN
El presente trabajo corresponde a un estudio etnográfico clásico, que tiene como objetivo explorar el espacio de construcción de los nuevos estilos de vida y prácticas corporales en los jóvenes skaters urbanos de Colima. El estudio se realizó en un parque emblemático: La Piedra Lisa, localizado en la capital del estado de Colima, México. La unidad de observación la integraron 50 jóvenes, entre 12 y 20 años, que practican el skate durante la semana. El método utilizado fue el etnográfico, las técnicas de investigación fueron la observación participante -durante un mes y medio-y la entrevista informal; y como instrumento de registro se empleó el diario de campo. En la sistematización de la información y clasificación se usó la Guía de Murdock. El estudio describe aspectos identitarios de los jóvenes skaters, información acerca del desarrollo de habilidades físicas coordinativas, condicionales y de experticia skate, lugares de encuentro y apropiación de espacios públicos, clasificación de los skaters por grupos, el equipamiento utilizado, y la percepción social que se tiene de sí mismos, de la sociedad, la familia y de los amigos.
\end{abstract}

Palabras clave: skate, juventud urbana, habilidades físicas, estilo de vida.

\begin{abstract}
The present work corresponds to a classic ethnographic study, which aims to explore the construction space of new lifestyles and bodily practices in young urban skaters in Colima. The study was conducted in an emblematic park: La Piedra Lisa, located in the capital of the state of Colima, Mexico. The observation unit was made up of 50 young people between 12 and 20 years old, who skate during the week. The method used was ethnographic, the research techniques were participant observations -for a month and a half- and the informal interview, and the field newspaper was used as recording instrument. For the systematization of information and classification, the Murdock Guide was used. The study describes identity aspects of young skaters, information about the development of coordinative, conditional and skateboarding physical skills, meeting places and appropriation of public spaces, classification of skaters by groups, the equipment used, and the social perception that it has of itself, society, family and friends.
\end{abstract}

Key words: skate, urban youth, physical abilities, life styles.

\footnotetext{
* Universidad de Colima, Colima, México.

** Secretaría de Educación, Mexicali, Baja California, México.
} 
Salazar C., C. M., Manzo L., E. G., Arellano C., A. C., Barajas P., L. T., \& Valdivia, A. (2020). Estudio etnográfico de jóvenes skater. prácticas corporales y urbanas. Revista Ciencias de la Actividad Física UCM, № 21(2), julio-diciembre, 1-12. DOI: http://doi.org/10.29035/rcaf.21.2.6

\section{Planteamiento del problema}

En los últimos 20 años, los estudios sobre la juventud evidencian que este grupo social es uno de los más vulnerables, ya que presentan distintas problemáticas, como: elevadas tasas de migración, ausencia de oportunidades de empleo bien remunerado, baja calidad educativa, niveles de escolaridad inferiores, inexistencia de espacios de recreación, influencia inadecuada de medios masivos de comunicación, incremento de adicciones, aumento de enfermedades de transmisión sexual y un alto número de embarazos a temprana edad (Lemus, 2005, Fernández \& Ávila, 1999), problemáticas sociales que los expone a diferentes situaciones de riesgo (Arellano, 2014).

Es necesario mencionar que, en la actualidad mexicana, se vive un momento histórico: hoy, el grupo etario entre 12 y 29 años representa la tercera parte de la población total del país (Encuesta Nacional de Juventud, 2010). México es un país joven, en virtud de que la mitad de la población tiene menos de 25 años. En este sentido, es importante tener en cuenta que los jóvenes desempeñan un papel muy importante en el desarrollo social y económico de un país; por tanto, si la sociedad brinda orientación apropiada, además de oportunidades de desarrollo en áreas claves -como educación, salud, empleo y recreación- los jóvenes podrán transitar a la edad adulta y contribuir significativamente en el desarrollo de sus contextos sociales (Arellano, 2014).

En este tenor, la educación, salud, empleo y recreación son los principales activos para que los jóvenes alcancen su desarrollo profesional y humano; por ello, se requiere que los investigadores sociales prestemos atención a los escenarios en los que se encuentra este grupo de la sociedad, pues sólo así podremos comprenderlos e incidir en la mejora de sus condiciones de vida (Arellano, 2014). Hablar de los jóvenes es considerar una etapa de construcción y vivencia de emociones, aprendizajes y amistades, para cimentar la vida futura en sociedad.
Arribas \& González (1987) mencionan que estas experiencias también desencadenan dificultades, las cuales quedan -en la mayoría de las ocasionesal descubierto, sin apoyo u orientación por parte de la familia o programas gubernamentales (Medina, 2010 en Cortés \& Díaz, 2012). Esto da oportunidad a los jóvenes para iniciarse en la vida por sus compañeros de escuela, vecinos, familiares de la misma edad, de tomar y apropiar conductas de protagonistas del espectáculo o de representantes de otras culturas juveniles. Y generando expresiones y comportamientos que salen de la norma de los adultos y del sistema social (Cortés \& Díaz, 2012)

Sin embargo, Morillo (2017) considera que, en la actualidad, esta etapa ha dejado de ser un periodo del ciclo vital para convertirse en un modo de ser que amenaza con envolver la totalidad del cuerpo social. Pareciera que la adolescencia y la primera etapa de la juventud estuvieran hoy institucionalizadas como una época de privilegios, con responsabilidades menores y tiempos bastante considerables para el ocio y la diversión (Guevara, 2017).

En el presente trabajo se plantearon tres objetivos: 1) explicar los beneficios del skate, 2) describir los usos y prácticas comunes de los jóvenes skater, y 3) explicar las percepciones sociales que tienen de sí mismos, de la sociedad, la familia y de los amigos, para, con ello, generar nuevas líneas de investigación que promuevan, a su vez, la generación de políticas públicas enfocadas a mejorar sus condiciones de vida.

\section{Marco teórico: El skate y los jóvenes}

El skate ha progresado, estructural y técnicamente, en un lapso de 50 años: de ser una práctica de jóvenes surfistas californianos en piscinas vacías ha transitado a una práctica corporal social, divergente y muy urbana, practicada por cientos de jóvenes. En 2020, el skate habrá logrado el objetivo de todo deporte alternativo: el acceso al olimpismo.

Para definir al skate se retomó la descripción de Carrillo, Guerrero \& Delgado (2011) quienes, desde 
Salazar C., C. M., Manzo L., E. G., Arellano C., A. C., Barajas P., L. T., \& Valdivia, A. (2020). Estudio etnográfico de jóvenes skater. prácticas corporales y urbanas. Revista Ciencias de la Actividad Física UCM, No 21(2), julio-diciembre, 1-12. DOI: http://doi.org/10.29035/rcaf.21.2.6

una perspectiva personal, como practicantes y jóvenes, la refieren de la siguiente forma:

El skateboarding no es sólo un juego, sino que es un deporte completo, los practicantes de este deporte dicen que el skate es una manera de expresión, al estar en el aire es como si volaran, el skate es completo porque favorece al sistema muscular de las extremidades inferiores, además que mejoran su elasticidad, el skate es una buena manera para bajar de peso, ya que con este deporte se queman muchas calorías ya que sudas mucho, se piensa que estar sobre la tabla no trabajas con ninguna parte del cuerpo, pero están equivocados ya que se necesita el equilibrio y para eso necesitamos fuerza en las piernas y en el abdomen para tener mayor control sobre la tabla, también se trabaja con las sensopercepciones, ya que necesitamos tener percepción espacio-temporal para poder esquivar los obstáculos del camino, las actividades en rampa (plano inclinado) requieren de una coordinación y mecánica especial, para resumirla, antes de bajar se visualiza por donde se desciende para evitar piedras o alguna basura, después impulsar la tabla con el pie y echar el cuerpo hacia delante y como a la a mitad de la rampa colocar tu cuerpo en la posición inicial para tener control, en esta pequeña síntesis podemos observar que se necesitan de varios componentes como la coordinación, el rito, la visualización, entre otros. Es importante considerar que es un deporte con sus riesgos, pero existen maneras de aplicar el skate sin tener que llegar a un nivel competitivo, sino que se puede trabajar de una manera segura y sencilla (pág. 14)

El gradiente y las expectativas sociales que se tienen sobre los jóvenes generan inquietudes juveniles que son expresadas por estos grupos, según el contexto social en el que se desenvuelven (Feixa, 1998); de esta forma, los jóvenes reproducirán estilos de vida que el medio les provea. Saraví (2010) menciona que las condiciones urbanas, propiciadas por las nuevas formas de convivencia social, ha rediseñado los comportamientos juveniles con nuevas y diferentes formas de autodefinirse (Lemus, 2005); por ello, la necesidad de hacer algo diferente que le motive y lo lleve al encuentro con sentidos corporales más significativos (Aguayo, 2003 en C. Salazar \& G. Manzo, 2019) y con un alto grado de adrenalina (Cortés \& Díaz, 2012); aunque el resto de la sociedad lo vea como pérdida de tiempo y adquisición de estilos de vida nocivos que, generalmente, entrará en el sentido de cambio y ruptura propio de las conductas juveniles frente a la tradición. Una actividad cíclica y generacional.

En los jóvenes, la experimentación -a través de sensopercepciones corporales- constituye la esencia de ser y existir. El cuerpo significa su conexión con todo lo que le rodea. En este sentido, Cachorro (2007, p. 48) considera que la construcción social está mediada por el cuerpo; por tanto, toda relación humana estará impregnada de referencia personalcultural:

el cuerpo es un objeto de referencia social y cultural, la posición del cuerpo, la manera de caminar, hablar, las gesticulaciones los movimientos corporales y un sin fin de manifestaciones expresadas que dan cuenta que el cuerpo es más que la encarnación del espíritu que interviene en la definición de los individuos y los grupos sociales.

Los estudios sociales recientes han generado conocimientos para comprender los nuevos modos de vivir el cuerpo y la construcción de experiencias y participación social de las comunidades. Bauman (2009) y Galindo (2013) hablan acerca de la nueva forma de visibilizar las sociedades para comprenderlas y estudiarlas; especialmente, las comunidades de jóvenes, quienes son los que han desencadenado nuevas formas de convivencia urbana. Bauman apela al concepto de sociedades líquidas, en donde nada es sólido, todo es cambiante; mientras que Jesús Galindo observa el fenómeno de las comunidades estéticas, caracterizadas por no tener 
Salazar C., C. M., Manzo L., E. G., Arellano C., A. C., Barajas P., L. T., \& Valdivia, A. (2020). Estudio etnográfico de jóvenes skater. prácticas corporales y urbanas. Revista Ciencias de la Actividad Física UCM, № 21(2), julio-diciembre, 1-12. DOI: http://doi.org/10.29035/rcaf.21.2.6

una exclusividad. El estado líquido refiere al aspecto cambiante de los ambientes sociales, con referencia a la actualidad; en tanto que Galindo construye su visión de estética en cuanto a los gustos y preferencias que conducen a un grupo particularmente joven.

Los jóvenes expresan cotidianamente lo que piensan y prefieren, amparados en la impronta de las TICS, que dirige, modela y confronta. En el caso de las prácticas corporales, los jóvenes han abandonado las actividades rígidas que no permiten la adaptación rápida e incorporación sencilla; ahora buscan patrones de adaptación menos frustrantes que luego les provocaría desertar por no cumplir con sus exigencias. Por ello, los jóvenes de esta generación se acercan a grupos interculturales y diversos, como los colectivos de skate (González, 2018).

Villagrán \& Scarnatto (2011) retoman estas posturas en sus trabajos con sujetos urbanos practicantes de parkour, skate (Cortés \& Díaz, 2012, Freeman \& Riordan, 2002, Camino, 2008, Márquez \& Díez, 2013, Moore, 2013) y breakdance. Han observado que los jóvenes tienen gustos y crean sentidos muy específicos de sus prácticas corporales; de agrupación y socialización, e ideado sus marcos de legitimidad -modos de romper o acordar con la tradición-, sus desplazamientos, sus sentimientos, sus movimientos y alternativas necesarias para sobrevivir la etapa joven.

La juventud representa -en un esquema tradicional de la sociedad occidental- un periodo de cambio, una ruptura con el mundo adulto, el espacio biológico y cultural donde se generan los grandes cambios sociales. Es el mundo juvenil, el inicio de la transformación humana: la ruptura, la brecha generacional es ya algo cíclico, en cada generación aparece este cambio; es una relación cerrada entre la formación del individuo en cuanto a las exigencias de la comunidad y las exigencias propias de su persona, para figurarse como tal entre sus haceres y el significado de sí mismo:
La acción es, a la vez, cierta configuración de movimientos físicos y una realización susceptible de ser interpretada en función de las razones de actuar que la explican [...] según la relación que prevalezca entre el concepto de persona y de cuerpo, y que obliga a decir que las personas son también cuerpos (Ricoeur, 2003, p.74).

Galindo (2013) estipula que los jóvenes, al no encontrar espacios de construcción identitaria flexibles y de acceso rápido, han tenido que ocupar espacios públicos, parques o calles de la misma ciudad; o bien, encontrar formas de vivir su etapa juvenil en los diversos contextos urbanos para expresar sus sentimientos y gustos (Lemus, 2005).

Estas emergencias juveniles ahora están conformándose y exhibiéndose corporalmente en lugares que antes no acostumbraban; las comunidades estáticas tienen como móvil expresar y ser el centro; ahora, transitan y se instalan en zonas vibrantes, públicas y socialmente activas, han dejado de ser tradicionales y estáticos.

En el caso de la práctica física, a los jóvenes urbanos ya no les satisface la tradición deportiva: es rígida, normada y basada en la competencia (Villagrán \& Scarnatto, 2011), en la actualidad, imponen una forma de práctica de actividad física que energetiza, les proporciona placeres corporales que hace a las sensaciones coordinativas, neuromusculares una prolongación del cuerpo, los vuelve vibrantes y les proporciona presencia en el paisaje urbano (Cachorro, 2012).

Por tanto, el trabajo que aquí se presenta pretende explorar el espacio de construcción de los nuevos estilos de vida y prácticas corporales en los jóvenes urbanos de Colima, desde la experiencia y vida de los jóvenes colimenses que practican Skate, para conocer el uso y la percepción que poseen de su actividad con respecto a la noción de ciudad. 
Salazar C., C. M., Manzo L., E. G., Arellano C., A. C., Barajas P., L. T., \& Valdivia, A. (2020). Estudio etnográfico de jóvenes skater. prácticas corporales y urbanas. Revista Ciencias de la Actividad Física UCM, № 21(2), julio-diciembre, 1-12. DOI: http://doi.org/10.29035/rcaf.21.2.6

\section{Diseño Metodológico}

\section{Tipo de estudio}

Por las características del estudio, se requieren datos empíricos, obtenidos de primera mano; es por ello que el método etnográfico -inmerso en un marco cualitativo- permite tener una observación activa en la recolección de datos, "la observación participante se refiere a situaciones en las que el investigador realmente participa en las costumbres y actividades de la sociedad" (Adams, 1971, p. 28). En este caso, se observó a los jóvenes que practican el skate en La Piedra Lisa.

\section{Población}

Fue necesaria una selección representativa de los skatos; para tal efecto, se realizaron charlas informales dirigidas a los más de 50 jóvenes (entre 12 y 20 años); entre ellos, 15 informantes clave "agentes informadores confiables, deseosos de comunicar lo que saben de su comunidad" (Pelto, 1975, p. 67), para obtener los datos de viva voz de los sujetos del estudio.

\section{Técnica}

El instrumento utilizado para el levantamiento de información fue el diario de campo; en este se registró lo que se observó, dialogó y describió del trabajo de campo; en otras palabras, se registra "una descripción exacta" (Malinowski, 1995: 20) de lo que se vivió desde el primer momento. Después de nutrir todos los días el diario de campo, la tarea siguiente consistió en sistematizar la información. A continuación, se llevó a cabo la descripción ordenada y clasificada de los datos, bajo la Guía de Murdock, que tiene el objetivo de "Clasificar con criterio sistemático los datos en existencia, de tal manera que se haga posible y fácil en cualquier momento encontrar la información necesaria para el estudio de una cuestión determinada" (Murdock, 1989, p.1).

Otra de las técnicas utilizadas en el proceso de recolección fue la entrevista informal, entendida como la máxima interacción personal posible entre el sujeto investigado y el sujeto investigador; así, se produce -en principio- en la situación de la llamada entrevista abierta (diálogo de persona a persona). Puede ser apoyado, por un "aparato técnico" que consiste en el sometimiento del sujeto investigado a escalas de actitudes e, incluso, determinadas situaciones psicodramáticas (Ricoeur, 2003). Sin embargo, en la investigación fue muy utilizada la entrevista individual abierta, la cual tiende a resultar altamente productiva para el estudio de casos típicos o extremos; pero, sobre todo, para revelar las relaciones de la identidad personal con los modelos culturales de personalidad-reflejados en el otro, generalizado o superego social-que conforman el ideal del entrevistado.

Esta técnica de investigación adopta, con frecuencia, la forma de encuesta de opiniones y actitudes, se articula mediante intercambios verbales entre entrevistador y entrevistado; $y$, a veces, tiende a limitarse prácticamente a registrar y procesar lo que el sujeto entrevistado dice: produce respuestas verbales a preguntas verbales (respuestas a cuestiones fácticas, como la edad del entrevistado o su situación laboral como trabajador), creencias, valores y fines del sujeto hablante, escapan en su nivel máximo de profundidad y articulación (Ortí, 1989).

\section{Resultados}

Los hallazgos que a continuación se presentan derivan de la convivencia y uso de las técnicas e instrumentos -anteriormente mencionados- que permitieron la documentación cotidiana en el diario de campo y posteriormente, categorizado en tres grandes temas con apoyo de la Guía de Murdock (guía para la clasificación de los datos culturales): los beneficios del skate, los usos y prácticas comunes, y las percepciones sociales.

\section{Beneficios físicos del skate}

Cuando nos referimos a un beneficio estamos hablando de un bien que se recibe físicamente por practicar un deporte como el skate. A continuación, 
Salazar C., C. M., Manzo L., E. G., Arellano C., A. C., Barajas P., L. T., \& Valdivia, A. (2020). Estudio etnográfico de jóvenes skater. prácticas corporales y urbanas. Revista Ciencias de la Actividad Física UCM, № 21(2), julio-diciembre, 1-12. DOI: http://doi.org/10.29035/rcaf.21.2.6

se explican seis aportaciones físicas que tiene la práctica de este deporte:

a) Equilibrio: es una de las principales capacidades desarrolladas a través del skate. Se adquiere por el hecho de subirse y conducir la tabla sin caerse en una cierta distancia y, rutinariamente, provoca el dominio del equilibrio. El desarrollo de las habilidades coordinativas es un hecho en este deporte; los skaters depositan en sus piernas y el balance de sus brazos el equilibrio de cada uno de los trucos. Van y vienen, tantean y lo mejoran a cada intento.

b) Coordinación: para los diferentes movimientos -cambio de pierna, giro de tabla en el momento adecuado, doblar y estirar- se potencia la fuerza de los músculos en las extremidades inferiores.

c) Resistencia: en combinación con la habilidad anterior, la resistencia juega un papel importante en esta práctica; los recorridos o el tiempo que le dedican estos jóvenes a la misma va logrando la adquisición de resistencia y, por último, la destreza para el logro de nuevos trucos.

d) Riesgos físicos: el elemento contradictorio en esta práctica es que, como consecuencia de una falta de disciplina, higiene corporal o de un especialista que guíe el calentamiento previo, puede ocasionar lesiones corporales a los practicantes de este deporte. Aunque, de acuerdo con algunos de los jóvenes observados, muchos se desplazan desde sus domicilios hasta el lugar donde se concentran patinando y esto prepara al cuerpo para el movimiento del skate. Sin embargo, otros tienen que abordar el transporte urbano, por lo tanto, la preparación física previa no es generalizada con los riesgos que esto conlleva.

e) Composición corporal: una particularidad de los jóvenes skatos que conforman el grupo de observación es la delgadez, a diferencia de los que patinan y andan en bicicleta. Aunque en el parque se subdividan en grupos, es notable la ausencia de obesidad; inclusive, los chicos skate se ubican mayormente en bajo peso.

f) Gasto energético: en esta actividad es notable que la mayoría camina/patina para llegar al parque, el resto combina con transporte público ("ruta" o taxi). Duran, aproximadamente, entre una y dos horas patinando, con espacios de descanso no mayores a tres minutos, tiempo que dedican para tomar un respiro, aminorar molestias/dolores y charlar con los otros skaters. El gasto energético por la actividad puede estar calculado entre 250-400 calorías por práctica, por el tipo de ejercicios que realizan, con ascensos y saltos.

\section{Usos y prácticas comunes}

El uso es la manera en la que los jóvenes emplean el skate, y la práctica está asociada a la regularidad con la que se realiza este deporte. En este sentido hablamos de usos y prácticas comunes, es decir, aquellas que se practican entre los jóvenes skater.

a) Zona de práctica skate: la Rotonda de los Colimenses Ilustres en el parque de "La Piedra Lisa".

El parque de la Piedra Lisa es un lugar tradicional para la recreación de los habitantes de la ciudad de Colima, situado sobre una calzada pletórica de vegetación y muy cercana al centro de la capital del estado; resulta un punto de reunión idóneo para el esparcimiento y la práctica de distintas actividades al aire libre. Posee un área de pequeños restaurantes típicos y de comida rápida, al lado de otra muy amplia (dedicada a los juegos infantiles); al fondo se encuentran canchas para futbol y baloncesto.

En fechas recientes se implementaron algunas transformaciones que provocaron una ruptura con la tradición: el gobierno estatal decidió la construcción de un museo interactivo -cuya infraestructura es planteada para los infantes- y un monumento dedicado a la memoria de hombres y mujeres de origen colimense que hayan aportado al desarrollo 
Salazar C., C. M., Manzo L., E. G., Arellano C., A. C., Barajas P., L. T., \& Valdivia, A. (2020). Estudio etnográfico de jóvenes skater. prácticas corporales y urbanas. Revista Ciencias de la Actividad Física UCM, N 21(2), julio-diciembre, 1-12. DOI: http://doi.org/10.29035/rcaf.21.2.6

e historia del lugar: la Rotonda de los Colimenses Ilustres. Precisamente, sobre esta explanada que, al fondo (a manera de altar), se resguarda con una serie de columnas -una para cada personaje ilustre-y que posee rampas laterales, lo cual facilita el acceso a dicha sección, se ha constituido el centro de reunión para los jóvenes practicantes del skateboard en esta ciudad. La imagen resulta contrastante, pues en un sitio dedicado a la historia, es decir, al pasado, con toda la quietud que dicha finalidad ofrece, aparecen los jóvenes en movimiento sobre sus patinetas: se deslizan, suben y bajan por las rampas, giran en la explanada frontal. En su mayoría, hombres, parece que vuelven exclusivo ese lugar para esta práctica corporal que innova en el transcurrir costumbrista de esta provincia mexicana.

b) Clasificación de los grupos skates del parque "La Piedra Lisa". Los agrupamientos surgen de acuerdo a la edad:

Grupo 7. Jóvenes de 12 a 15 años, con seis integrantes. Realizan trucos de bajo impacto, como es el caso de Ollie y Kickflip; se caracterizan por una apariencia tranquila y sana con elementos de bajo grado de dificultad, con ritmo relajado, con más tiempo de descanso que aprovechan para hidratarse o charlar con sus amigos.

Grupo 2. En un rango que va de 16 a 19 años, es el grupo más numeroso: con un aproximado de 17 integrantes; la mayoría posee un tiempo considerable patinando. Puede considerarse que manejan un grado de dificultad medio, realizando los siguientes elementos: Indy, Melon, Behinana, Roastbeef, Christ Air, Nosegrae. Algunos, ya con un dominio de alto grado en los elementos Axle Stall, Nose Stall, Rock to Fakie, Rock 'n' Roll, Boneless, Invert, Handplant. Este grupo dedica más horas a la práctica, menos tiempo de reposo y más constancia en su asistencia diaria.

Grupo 3. Son los jóvenes de 19 a 21 años, constituido por cinco jóvenes que patinan de una forma más ligera y rápida con técnicas particulares de ellos mismos, con el menor tiempo posible de reposo; no realizan tantos trucos, se centran más en sólo patinar con una velocidad mayor. Un dato curioso de estos jóvenes es que no siempre acuden todos, ni a la misma hora, por las diferentes ocupaciones de escuela y/o trabajo.

c) Tiempo de experiencia: a través del diálogo con los jóvenes skate se obtuvo la información acerca de su tiempo de experiencia hacia esta práctica. Se organizaron tres grupos, atendiendo la duración de su práctica: 1) Jóvenes adolescentes con uno a tres meses patinando. 2) Aquellos cuya experiencia rebasa los tres meses hasta un año. 3) Sujetos con uno hasta tres años.

d) Vestimenta de jóvenes skate: la mayoría de los jóvenes usa el mismo estilo de ropa; pantalón de mezclilla stretch (pegado al cuerpo), los colores más dominantes son azul, negro y café; la camisa es holgada, preferentemente lisas y de marcas comerciales extranjeras. Otro rasgo muy particular es la gorra, y los que no la usan, el corte de pelo es largo y bien peinado. De igual forma, acuden y patinan con una mochila pequeña en su espalda y audífonos. A diferencia de otras disciplinas consideradas como deporte, la vestimenta no requiere especificaciones: con la misma ropa se puede ir a patinar como a cualquier otro compromiso.

e) Gustos, aficiones y comportamientos de los jóvenes skate.

En su forma de hablar utilizan palabras propias del argot juvenil: pendejo, cabrón, güey, puesto que se encuentran en la postura de ruptura de reglas y en plena libertad. La mayoría fuma sin considerar consecuencias; para ellos es parte del estilo skate. La personalidad de estos chicos raya en el egocentrismo, no molestan a los demás, son serios, intensos en sus expresiones; en otros casos, distraídos; algunos manifiestan poco interés por la escuela, son sencillos. Aparecen también en el extremo: 
Salazar C., C. M., Manzo L., E. G., Arellano C., A. C., Barajas P., L. T., \& Valdivia, A. (2020). Estudio etnográfico de jóvenes skater. prácticas corporales y urbanas. Revista Ciencias de la Actividad Física UCM, N 21(2), julio-diciembre, 1-12. DOI: http://doi.org/10.29035/rcaf.21.2.6

prepotentes, groseros y hasta grotescos. Gustan de la música hip-hop, rap, del reggae; ya que se sienten identificados con las letras o las personalidades de los artistas como Bob Marley o Shaggy.

f) Equipamiento y usos de este. De acuerdo a lo observado, se utilizan diferentes estilos de tablas: -Longboards. Se utilizan para bajar cuestas, ya sea en carreras, en slalom o, simplemente, como medio de transporte. Al ser más ancho, otorga más estabilidad, seguridad y comodidad que una patineta normal. Su tamaño y peso los hace menos apropiados para monopatines de trucos.

- Tablas de Street. Están preparadas con más láminas de madera que las normales para evitar roturas inminentes de la tabla y poseen unos ejes más recubiertos de metal para facilitar su deslizamiento por superficies ásperas. Éstas, necesitan una especial dureza en sus ejes, debido a que en las caídas requieren mayor soporte; es necesario aumentar la dureza a las ruedas para evitar su deformación mediante golpes abruptos a sus laterales; o en manuales, como rail switch flip half flip que, durante el aterrizado-después de un salto de obstáculos- puede deformarlas bastante; o con el to rail handstand side shovit, que su particular posición puede llegar a deformarlas muy significativamente.

-Tablas de tricks. Son las tablas especiales para skate parks, ya que pesan un poco más, pero son las ideales para hacer giros y moverse por rampas empinadas.

g) Destrezas y trucos. Las habilidades específicas que los practicantes desarrollan se denominan trucos, como si fueran efectos de la magia; provocan que el dominio del espacio, la velocidad y la especialización de los movimientos intervengan en movimientos que sólo se logran a través de la experiencia y la dedicación.
En el desarrollo de los trucos es muy importante la colocación de los pies en la patineta: si se lleva el pie izquierdo delante se dice que ese skater es "normal"; mientras que si el que va delante es el derecho se dice que es "goofy". Esto condiciona la realización de los trucos y la posición llamada "switch" mide la dificultad del dominio en los pies.

El truco más básico es el ollie, que es el salto que se da con el monopatín; cuando algún truco se hace yendo marcha atrás se dice que se ha hecho de fakie. Al girar en el aire, si se hace hacia adentro, "dando la espalda", es backside; mientras que hacia afuera, "dando la cara", se dice que es de frontside.

\section{Percepciones sociales}

La percepción social la comprendemos como la manera en que se percibe y se valora el skate. En ese sentido fue importante conocer la forma en que la familia, la comunidad, los amigos y los propios skater se ven y se valoran.

a) De la familia: algunos reciben apoyo de sus padres, la aceptación transita de un pasatiempo hasta deporte. En otros casos los padres consideran esta actividad una pérdida de tiempo, los chicos son calificados como "vagos" y carecen de apoyo familiar.

b) La comunidad de la Piedra Lisa: al conversar con algunas personas visitantes del parque, la aceptación es total. Han cedido el espacio central del parque, al considerar que "es mejor verlos disfrutar de un pasatiempo, se ejercitan y no hacen daño a nadie [Señora de 35 años]". Sin embargo, a otro sector que no precisamente asiste al parque, el comportamiento de estos jóvenes no dista más allá de una pérdida de tiempo, reunión de "vagos sin oficio" y mala imagen al espacio público.

c) Los iguales (amigos): al cuestionarles acerca de la percepción que tienen los amigos de su práctica skate, reciben discursos positivos; la mayoría perciben la actividad como algo novedoso e 
Salazar C., C. M., Manzo L., E. G., Arellano C., A. C., Barajas P., L. T., \& Valdivia, A. (2020). Estudio etnográfico de jóvenes skater. prácticas corporales y urbanas. Revista Ciencias de la Actividad Física UCM, № 21(2), julio-diciembre, 1-12. DOI: http://doi.org/10.29035/rcaf.21.2.6

interesante. Cabe destacar que los skaters han hecho amigos en los parques donde patinan.

d) Autopercepción y vivencias desde y con el skate: cada persona habla de un proceso distinto, una manera particular de vivir esta práctica corporal, desde donde podemos especular construcciones ubicadas entre lo deseable y lo posible; donde la verdad, muchas veces, tendrán que juzgarse desde la óptica de un mundo experiencial que aún resulta limitado. Se extractan algunas historias personales contadas por los jóvenes:

i) Esto es lo mejor porque no tiene un horario fijo establecido, y no es una práctica excluyente, agobiante o tediosa, que en el momento que empieza a patinar se olvida de todo y tan sólo existe él y el skate.

ii) El skate es una moda o nueva tendencia dicen [los patinadores] que el skate, les ha brindado popularidad y visibilidad, ha logrado conocer amigos y chicas, ha pasado a ser su estilo de vida y un escape para no estar en casa.

iii) "Mi mamá está en Estados Unidos y la veía poco, vivo con mi papá, pero me presta poca atención e interés, mi conducta... no le importa a qué horas llegue o que actividades haga durante el día, ni que fumo mariguana, él me dice que desde los 16 años consumía mariguana [el padre] y que, por lo tanto, lo traía en la sangre" [skater].

iiii) "Estuve en la cárcel dos años, soy casado y tengo un hijo; para mí, skate representa una cultura, forma de desafiar al gobierno y romper con sus reglas en los espacios públicos para darme a notar" [skater].

\section{Discusión}

La actividad física del skate - de acuerdo a lo observado- constituye un proceso de reapropiación del espacio donde habita el joven urbano. La ciudad, un símbolo de modernidad y evolución, tiende a disminuir la actividad física; por lo tanto, esta ubicación de los grupos para el skate puede ser el resultado de la búsqueda de los jóvenes para alcanzar cierto grado de autonomía para su corporeidad. El movimiento, más allá de una serie de acciones realizadas por el cuerpo, es una abstracción del espacio y la relación del cuerpo humano con los objetos del contexto.

Entre los beneficios que perciben, más allá del logro físico-biológico, asumen una riqueza social que propicia el encuentro con otros y con otras en iguales circunstancias (problemas familiares, escasez alimentaria y consumo de sustancias ilegales). Entre los hallazgos más relevantes del estudio se observan subdivisiones de grupo, basadas en la habilidad física, estilo deportivo, nivel socioeconómico, género y grupo de amistades; los skatos urbanos, mayores de 15 años, han encontrado en esta práctica el medio de estilización personal y expresión de frustraciones, resentimientos y contradicciones propias de la edad; los skatos más chicos fortalecen su autoconfianza al mejorar sus destrezas físicas.

La personalidad del skater tiende hacia el logro individual; situación que refleja las características del equipamiento. La tabla está diseñada para una sola persona, por lo tanto, los movimientos involucran las extremidades inferiores y la baja espalda. Si bien tienden a agruparse para la práctica de rutinas, más que sentirse cuestionados socialmente, lo hacen para señalar un territorio que les asegure el libre tránsito de su tabla.

El agrupamiento de los practicantes de esta modalidad de movimiento hace visible el sentido de organización que la sociedad actual ofrece para los jóvenes, ya que el enfrentamiento generacional es un elemento cíclico: cada época presenta la separación entre lo nuevo y lo anterior. Pareciera que no hay tal rompimiento, sino el proceso de transformación que implica el renacer social. El joven skater siente, se autodenomina "rechazado" en la mayoría de los casos; pero falta información al respecto en un estudio donde se impliquen las historias de familia y la entrevista en profundidad, por citar un ejemplo. La separación con el adulto tendrá las consecuencias que cada familia o núcleo social señale 
Salazar C., C. M., Manzo L., E. G., Arellano C., A. C., Barajas P., L. T., \& Valdivia, A. (2020). Estudio etnográfico de jóvenes skater. prácticas corporales y urbanas. Revista Ciencias de la Actividad Física UCM, No 21(2), julio-diciembre, 1-12. DOI: http://doi.org/10.29035/rcaf.21.2.6

como pertinente al código ético con el que se desenvuelva.

\section{Conclusión}

De esta forma, la práctica del skate en la ciudad de Colima refleja un espacio de oportunidad para sistematizar la actividad juvenil que, a su vez, genere propuestas productivas y pertinentes para este sector de la población que se encuentra supeditado a imaginarios sociales y operaciones institucionales.

Se constituye en una zona de oportunidad para apreciar las distintas modalidades que surgen en las manifestaciones de las agrupaciones juveniles, lejos ya del sentido de banda, de la degradación y manipulación social; así, transformado el concepto, ser joven en una provincia mexicana implica la búsqueda de opciones para una vida plena que se vuelve del yo hacia el nosotros, en un estado de transformación permanente.

\section{REFERENCIAS BIBLIOGRÁFICAS}

Arellano, A. (2014). Representaciones sociales que los jóvenes de Colima tienen sobre su proyecto y expectativas de vida. Un estudio a partir de su vida cotidiana (Tesis doctoral). Universidad de Colima, Colima, México.

Arribas Macho, J., \& González Rodríguez, J. (1987). La juventud de los ochenta. Estudio sociológico de la juventud de Castilla y León. VaIladolid: Junta de Castilla y León. Consejería de Cultura y Turismo.

Adams, N. (1971). Un comentario sobre métodos antropológicos. Human Organization, 12(2), 10-14.

DOI: https://doi.org/10.17730/humo.12.2.u6710656 $473 m 2502$

Bauman, Z. (2009). Modernidad Líquida. Buenos Aires: Fondo de Cultura Económica.
Cachorro, G. (2007). Cuerpo y subjetividad: materiales y tensiones. Oficios Terrestres. Buenos Aires: Universidad Nacional de la Plata.

Cachorro, G. (2012). Memorias del cuerpo en la ciudad. Capitales corporales. XIV Congreso RedCom. Investigación y extensión en comunicación: sujetos, políticas y contextos. Congreso llevado a cabo en La Plata, Argentina.

Camino, X. (2008). Reinterpretando la ciudad: la cultura skater y las calles de Barcelona. Apunts Educación Física y deportes, (91), 54-65. Recuperado de https://www.raco.cat/index.php/ApuntsEFD/article/view/300074

Carrillo, J., Guerrero, K., \& Delgado, J. (2011). Percepción de los atletas, jóvenes y adultos de Villa de Álvarez y Colima respecto al skateboarding (Tesis de Grado). Universidad de Colima, Colima, México.

Cortés, E., \& Díaz, P. (2012). Visibilidad de las expresiones juveniles en Toluca. En E. Cortés, \& E. López, Expresiones... sentidos de vida en jóvenes (pp. 53-76). México: INDECUS.

IMJUVE, S. (2010). Encuesta Nacional de Juventud 2010. Instituto Mexicano de la Juventud y Centro Regional de Investigaciones Multidisciplinarias CRIM - UNAM. Recuperado de https://www.gob.mx/imjuve/documentos/base-de-datos-de-la-encuesta-nacional-de-juventud-2010

Feixa, C. (1998). El reloj de arena. Culturas juveniles en México. México: SEP/Causa Joven.

Fernández, H. \& Ávila, G. (1999). Análisis comparativos entre jóvenes rurales y urbanos. Jóvenes, Revista de Estudios sobre Juventud, cuarta época, (9).

Freeman, C., \& Riordan, T. (2002). Locating skateparks: The planner's dilemma. Planning Practice and Research, 17(3), 297-316. DOI: https://doi.org/10.1177/1206331208320488 
Salazar C., C. M., Manzo L., E. G., Arellano C., A. C., Barajas P., L. T., \& Valdivia, A. (2020). Estudio etnográfico de jóvenes skater. prácticas corporales y urbanas. Revista Ciencias de la Actividad Física UCM, № 21(2), julio-diciembre, 1-12. DOI: http://doi.org/10.29035/rcaf.21.2.6

Galindo, J. (2013). Comunidades estéticas e ingeniería en comunicación social. De los mundos emergentes. El caso del movimiento\# Yo soy 132. Revista de Estudios para el Desarrollo Social de la Comunicación, (8), 153169.

González, R. (2018). La comunicación visual en la cultura del patinaje extremo y su influencia en los jóvenes de Guayaquil Skateboarding Club (Tesis de Licenciatura). Universidad de Guayaquil, Guayaquil, Ecuador.

Guevara, Y. (2017). Expresión, manejo y reconocimiento de emociones y la valoración del barrio en integrantes de tribus urbanas que practican skateboarding de Trujillo, 2016. (Tesis de Licenciatura). Universidad Privada del Norte. Trujillo, Perú. Recuperado de https://repositorio.upn.edu.pe/handle/11537/13622?show=f ull

Lemus, G. (2005). Rapy prácticas de resistencia: una forma de ser joven. Reflexiones preliminares a partir de la interacción con algunas agrupaciones bogotanas. Tabula Rasa, (3), 253-270. Recuperado de http://revistatabularasa.org/numero-3/castiblanco.pdf

Márquez, I., \& Díez, R. (2013). La cultura skate en Madrid: una aproximación etnográfica. XI Congreso de Sociología. Federación Española de Sociología. Madrid, España. Recuperado de https://www.academia.edu/4132086/_La_cultura_skate_en_Madrid_una_aproximaci\%C3\%B3n_etnogr\%C3\%A7fica_junto_con_Israel_V_Marquez_XI_Congreso_Espa\%C3\%Blol_de_Sociolog\%C3\%ADa_Comunicaci\%C3\%B3n_del_Grupo_Sociolog\%C3\%ADa_de_la_Cultura_y_de_las_Artes_Madrid_UCM_julio_de_2013
Malinowski, B. (1995). Los argonautas del Pacífico occidental, comercio y aventura entre los indígenas de la Nueva Guinea Melanésica (4a Ed.). Barcelona: Península.

Moore, L. (2009). An Ethnographic Study of the Skate boarding Culture. The sport Journal (21). Recuperado de https://thesportjournal.org/article/an-ethnographic-study-ofthe-skateboarding-culture/

Morillo, J. L (2017). Rasgos adolescentes de la posmodernidad. Recuperado de https://independent.academia.edu/Jos\%C3\%A9Mori1104

Murdock, G. (1989). Guía para la clasificación de los datos culturales. Iztapalapa, Ciudad de México: Universidad Autónoma Metropolitana. Recuperado de https://docer.com.ar/doc/xxxn10

Ortí, A. (1989). La apertura y el enfoque cualitativo o estructural: la entrevista abierta semidirectiva y la discusión de grupo. En M. García Ferrando, J. Ibáñez, \& F. Alvira, El análisis de la realidad social. Métodos y técnicas de investigación (pp. 171-203). Madrid: Alianza.

Pelto, P. (1975). El estudio de la antropología. México: Unión Tipográfica Editorial Hispano Americana.

Ricoeur, P. (2003). Sí mismo como otro. México: Siglo $X X I$.

Roman, C., Villagrán, J., \& Scarnatto, M. (2011). Itinerarios del cuerpo y la ciudad. El caso del parkour en la Ciudad de Plata. IX Congreso Argentino y IV Latinoamericano de Educación Física y Ciencias. Universidad de la Plata. Congreso llevado a cabo en La Plata, Argentina. Recuperado de http://sedici.unlp.edu.ar/handle/10915/34205

Salazar, C., \& Manzo, G. (2019). Contexto social y urbano en los hábitos de actividad física deportiva de jóvenes de una población rural de México. Revista Edu-Física, 77(24), 93-103. 
Salazar C., C. M., Manzo L., E. G., Arellano C., A. C., Barajas P., L. T., \& Valdivia, A. (2020). Estudio etnográfico de jóvenes skater, prácticas corporales y urbanas. Revista Ciencias de la Actividad Física UCM, N²1(2), julio-diciembre, 1-12. DOI: http://doi.org/10.29035/rcaf.21.2.6

Recuperado de https://www.researchgate.net/publication/340362452_Contexto_Social_y_urbano_en_los_habitos_de_actividad_fisica_deportiva_en_jovenes_de_una_poblacion_rural_en_Mexico

Salazar, C., Manzo, E., \& Medina, R. (2013). Influencia de la vida moderna en la hipo-actividad física. En P. Reynaga-Estrada, \& V. Muñoz Fernández (Coord.), Actividad física y salud en la formación del profesional de la cultura física y del deporte. México: Universidad de Guadalajara.

Saraví, J. R. (2010). Los y las jóvenes que practican skate en la Ciudad de La Plata: presentación del proyecto de Investigación. IV Jornadas de investigación en Educación Corporal. Universidad de la Plata. Jornadas Ilevadas a cabo en La Plata, Argentina. Recuperado de http://www.memo-

ria.fahce.unlp.edu.ar/trab_even-

tos/ev.903/ev.903.pdf

UNICEF. (2012). Estado Mundial de la Infancia 2012. Niñas y niños en un mundo urbano. Nueva York: UNICEF.

Valles, M. (2000). Técnicas cualitativas de investigación social. Madrid: Síntesis. Recuperado de http://mastor.cl/blog/wp-content/uploads/2011/12/Tecnicas-CualitativasDe-Investigacion-Social-Valles.pdf

Villagrán, J., \& Scarnatto, M. (2011). Itinerarios del cuerpo y la ciudad. El caso del parkour en la Ciudad de Plata. IX Congreso Argentino y IV Latinoamericano de Educación Física y Ciencias. Universidad de la Plata. Congreso llevado a cabo en La Plata, Argentina. Recuperado de http://sedici.unlp.edu.ar/handle/10915/34205

Dirección para correspondencia

Dra. Ciria Margarita Salazar C.

Dra. en Educación Física, Universidad de Colima

Dirección postal: Luis Spota 39. Col. Morelos. Col. Colima, 28040.

Colima, México

ORCID: https://orcid.org/0000-0001-8863-2309

Contacto:

ciria6@ucol.mx

Recibido: 27-05-2020

Aceptado: 10-09-2020 